\title{
Influence of storage time and blending on irradiated sodium alginate-polyethylene oxide films modified by methyl acrylate monomer
}

\author{
Sumaia Aktar Sumi ${ }^{1} \cdot$ Md. Wasikur Rahman $^{1}$ (i) $\cdot$ Nirmal Chandra Dafader ${ }^{2}$
}

Received: 15 March 2020 / Accepted: 20 May 2020 / Published online: 1 June 2020

(c) Springer Nature Switzerland AG 2020

\begin{abstract}
The present work is an attempt to understand the effect of storage time on physico-mechanical properties of sodium alginate (SA)-polyethylene oxide (PEO) blended films modified by methyl acrylate (MA) monomer. The films were prepared by casting method and improved by $\gamma$ irradiation (12 kGy), PEO 10\%, Glycerol (Gol) 15\%, Mustard oil (MO) 20\% and MA $7 \%$ on a mass basis as optimized. The polymeric blend was stored for six months at room temperature. The physical and mechanical properties of the as-prepared SA-based films were investigated with $\gamma$ irradiation and compared the results obtained before and after the storage periods. The tensile strength (TS), elongation at break (EB), Young modulus (YM) and moisture content (MC) of the blended films were determined. Morphological and thermal features of the films were characterized by Scanning Electron Microscopy (SEM) and Dynamic Mechanical Analysis (DMA), respectively. Reaction scheme of the stored SA-based polymer films and causes of photodegradation of the films are explained in the context. The ultimate results of the present study showed remarkable enhancement in tensile properties $(>35 \%)$ and reduction in $\mathrm{EB}(\sim 30 \%)$ and $\mathrm{MC}$ of the films thanks to storage time and $\gamma$ irradiation.
\end{abstract}

Keywords Sodium alginate $\cdot$ Methyl acrylate $\cdot \gamma$-irradiation $\cdot$ Tensile properties $\cdot$ Storage time

\section{Introduction}

To preserve the physical and mechanical properties of polymeric materials toward practical application is a current issue. However, the polymers show adverse effect on complex processing as well as high production or recovery cost. Crosslinked polymers increase the life of blended films as well as workable properties owing to their closely packed molecular arrangement. They enhance the weather resistance for absorption of the fluids and provide a high tensile strength, compressive strength, bear and tear resistance to degradation. Cross-linked blends can be made by adding low molecular weight curing agents or monomers during the blend or coating formation and their properties can be improved through storage [1, 2]. Therefore, blending and storage of polymeric materials based on synthetic and natural polymers is a crucial concern for the 21st century.

Hygroscopic equilibrium of alginate depends on the amount of water contained in the relative humidity. Degree of polymerization (DP) and molecular weight and viscosity of sodium alginate solution is directly related to storage time. Alginates have wider spread application in the food and drink, pharmaceutical and bioengineering industries due to their remarkable gelation properties [3]. Sodium alginate (SA) (MW 10,000, viscosity 30.75 Cps at room temperature) [4], a polyelectrolyte having rigid molecular chain [5], and good film forming ability,

\footnotetext{
$\triangle$ Md. Wasikur Rahman, mwrahman.ump@gmail.com | ${ }^{1}$ Department of Chemical Engineering, Jashore University of Science and Technology, Jashore 7408, Bangladesh. ${ }^{2}$ Nuclear and Radiation Chemistry Division, Institute of Nuclear Science and Technology, Bangladesh Atomic Energy Commission, Dhaka 1207, Bangladesh.
} 
has extensively been exploited and studied in detail on biomedical applications as a drug carrier [6]. Sargassum (brown algae) is the main source of SA which is abundant in the Coral Island, St. Martin's in Bangladesh. Patel et al. [7] modified SA by carboxymethylation, as the modification enhances the behavior of SA toward grafting, due to the combined influence of the following factors: (a) carboxymethylated groups increase the swelling ability of $\mathrm{SA}$, thereby facilitating diffusion of the monomer and initiator, and (b) the ionization of carboxyl groups along the SA chains introduces negative charges which attract positive ions to the SA molecules leading to the formation of more active sites, available for the monomer, thus increasing the reactivity of SA.

Polyethylene oxide (PEO) (MW $300,000 \mathrm{~g} / \mathrm{mol}$ ), is a unique class of water-soluble, aerobically biodegradable thermoplastic $[8,9]$. Due to its excellent biocompatibility and very low toxicity, the potential use of PEO in biomedical applications has attracted a great deal of attention regarding both industrial and scientific points of view [10-12]. It has been reported that the primary hydroxyl group on cellulose and methylcelluloses can form a hydrogen bond to ether oxygen in PEO [13]. Similarly, hydroxyl groups on SA can also form a hydrogen bond to the ether oxygen in PEO. PEO as a suitable candidate blended with $S A$ was therefore selected.

Before monomer (MA) addition, the SA-PEO blends need to be tailored by plasticizer (glycerol) and emulsifier (mustard oil, MO) for suitable working environment of monomer. Plasticizer improves film flexibility and emulsifier provides uniform distribution of the monomer in film-forming suspensions. Hendrix et al. [14] showed that films prepared without glycerol (plasticizer) were brittle and cracked on the casting plates during drying. However, the addition of more glycerol than 33.3\% (dry basis) resulted in sticky and wet films. The concentrations of glycerol require to be optimized for forming the grafting polymer films (15-60\%) [15-18].

With a view to get the promising modifications of polymers, grafting has received much attention recently in which a variety of functional groups are imparted to a polymer by chemical treatment, photo-irradiation, high-energy radiation technique, etc. Graft polymers are segmented copolymers with a linear backbone of one composite and randomly distributed branches of alternative composite. Graft polymers have been synthesized for many decades and are especially used as impact resistant materials, thermoplastic elastomer, compatibilizers, or emulsifiers for the preparation of stable blends or alloys. To achieve desired properties of the SA-PEO films, further modification has been done by methyl acrylate (MA) and gamma irradiation [19].
It has been reported that storage has a great impact on the mechanical properties of polymeric film coats applied onto a dosage form. Here the driving factors are the amount of plasticizers added, types of plasticizers, film forming conditions, storage temperature and humidity. Changes in mechanical properties of the polymeric films may ultimately influence the drug release, stability and the final physico-chemical properties of the coated dosage forms [20]. To the best of our knowledge, SA-PEO blends with MA monomer addition grafted by $\gamma$ irradiation for long term storage periods were not studied so far.

The ultimate goal of the present work is to investigate the effect of storage time on mechanical and moisture absorption properties of irradiated SA-based films towards practical application. Particularly, the polymeric formulation was modified by blending with PEO, Gol, MO and MA monomer and irradiated with $\gamma$ followed by storage for six months. The film-forming additives were optimized and morphological and thermal features of the films were studied.

\section{Experimental}

\subsection{Materials}

Sodium alginate ( $M W \sim 200,000 \mathrm{~g} / \mathrm{mol}$ ) (Unichem, India), polyethylene oxide (MW $300,000 \mathrm{~g} / \mathrm{mol})$, methyl acrylate (Sigma-Aldrich, USA), glycerol (Sigma-Aldrich, India) and mustard oil (local market) were collected for the experiment and used as received.

\subsection{Procedure of SA-based film preparation}

Pure solution of SA of various percentages $(2,2.5,5,7$ and $10 \mathrm{wt} \%)$ was prepared. Then the solution was kept at rest for $1 \mathrm{~h}$ to remove bubbles by settling at room temperature followed by pouring the aqueous solution $(100 \mathrm{ml})$ onto a petri dish (diameter $10 \mathrm{~cm}$ and and a height to diameter ratio 1:6) to prepare thin films of almost uniform thickness $(\sim 4 \mathrm{~mm})$. The films were dried at $233 \mathrm{~K}$ in an oven under vacuum until they turned into transparent. Then the dried films were stored for characterization. A $2.5 \mathrm{wt} \%$ aqueous solution of SA was optimized for further processing. Similarly, various concentrations of PEO solution $(2,2.5,5$ and $7 \mathrm{wt} \%$ ) were prepared and tensile properties of the films were measured. A $2.5 \mathrm{wt} \%$ PEO showed higher TS and was considered for further study. SA and PEO blend was prepared by mixing formerly prepared $2.5 \mathrm{wt} \%$ aqueous solution of both SA and PEO in different compositions ( 0 , $5,10,20,30$ and $40 \mathrm{wt} \%$ PEO and balance SA). Then the films were characterized to optimize the composition of the blends. Best tensile properties showed for SA:PEO=9:1 
(w/w) blend film, considered for further experiment. SA-PEO-Gol blend was prepared by previously optimized $\mathrm{SA}: \mathrm{PEO}=9: 1(\mathrm{w} / \mathrm{w})$ and glycerol of various compositions $(0,10,15,20,25$ and $30 \mathrm{wt} \%)$. Then tensile properties of the films were studied to optimize the plasticizer composition and found best results for $15 \mathrm{wt} \%$ glycerol. So, further work was done with the blend composition (SA:PEO= 9:1 $(\mathrm{w} / \mathrm{w})$ and glycerol $15 \mathrm{wt} \%$. Similar procedure was followed for mustard oil of various compositions $(0,10,15,20,25$ and $30 \mathrm{wt} \%)$ and SA-based films containing MO (20 wt\%) showed optimum result. Optimum compositions of the final formulated samples were found to be $S A / P E O=90 / 10$ $(\mathrm{w} / \mathrm{w}), \mathrm{Gol}=15 \mathrm{wt} \%$ and $\mathrm{MO}=20 \mathrm{wt} \%$, would be applied in the additional investigations.

\subsection{Modification of the film with monomer and radiation}

The modification of the previously formulated blend was performed by soaking the blend in different formulations of MA $(0,3,5,7$ and $10 \mathrm{wt} \%$ and balance methanol-water solution $(\mathrm{v} / \mathrm{v}),(1: 1)$. Then some of the SA-PEO blends incorporated with glycerol, $\mathrm{MO}$ and $\mathrm{MA}$ were treated with $\gamma$ irradiation from ${ }^{60} \mathrm{Co}$ at the dose rate of $2.5 \mathrm{kGy} / \mathrm{h}$ in presence of oxygen. The dose rate was determined with the help of the Fricke dosimeter. The blends were irradiated with various radiation doses $(0,3,5,7,10,12,15$ and $20 \mathrm{kGy}$ ) and stored for six months in a desiccator at room temperature $\left(25 \pm 2{ }^{\circ} \mathrm{C}\right)$ and average relative humidity $\sim 78 \%$. Various properties such as tensile strength (TS), elongation at break (EB), Young modulus (YM) and moisture content $(\mathrm{MC})$ of the stored and non-stored films were measured and compared them.

The blends with different additives will be defined in this manuscript as capitalized first letters of each additive: $\mathrm{SA}+\mathrm{PEO}$ as $\mathrm{SP}, \mathrm{SA}+\mathrm{PEO}+\mathrm{Gol}$ as $\mathrm{SPG}, \mathrm{SA}+\mathrm{PEO}+\mathrm{Gol}+\mathrm{MO}$ as SPGM and $\mathrm{SA}+\mathrm{PEO}+\mathrm{Gol}+\mathrm{MO}+\mathrm{MA}$ as SPGMM

\subsection{Measurement of film properties}

The morphological analysis of the films was done by using scanning electron microscopy (SEM) of Model JEOL 6400 at an accelerating voltage of $15 \mathrm{kV}$. The SEM specimens were sputter coated with gold. The thermal properties of the films were studied by means of Dynamic Mechanical Analysis (DMA) (Triton Technology TTDMA, UK) from 28 to $200{ }^{\circ} \mathrm{C}$ at a heating rate of $4{ }^{\circ} \mathrm{C} / \mathrm{min}$ and an oscillating frequency of $1 \mathrm{~Hz}$.

Tensile properties of the films were measured by Testometric Rochdale England (DBBMTCL-250 kg) with a crosshead speed of $2 \mathrm{~mm} / \mathrm{min}$. The experimental load of $500 \mathrm{~N}$ and gauge length of $20 \mathrm{~mm}$ were used throughout the experiment. ISO 37-1977 (E) method was followed to evaluate tensile strength of the films. For the measurement of moisture content, the films were cut into $1 / 2 \mathrm{~cm}$ size. Then they were dried in an oven at $105^{\circ} \mathrm{C}$ and weighed successively for $1 \mathrm{~h}$ interval until the weight became approximately constant. Then the moisture absorption was calculated by the following equation:

Moisture absorption $(\%)=\left[\left(W_{m}-W_{d}\right) / W_{d}\right] \times 100$

where $W_{m}$ is the weight of the moisture absorbed and $W_{d}$ that of the dry films.

All experiments were carried out at room temperature $\left(25^{\circ} \mathrm{C}\right)$ and atmospheric pressure. At least three measurements were carried out for each experiment and data presented with standard deviation.

\section{Results and discussion}

\subsection{Morphological analysis}

The surface morphology of SPGMM blend before storage (Fig. 1a) and after storage (Fig. 1b) were investigated by using SEM. The surface morphology of both the SPGMM blends showed remarkable distinction properties. In the first case, the films exhibited relatively rough surface and the other image showed rather smooth surface pattern. This result might be the effect of storage period on the SA-based PEO blend films [21]. In fact, SA-based polymeric blends are tightly bound with various additives; however, there are some holes inside where some small sized fragmented particles are distributed (Fig. 1a). The holes could be due to the presence of uncross-linked polymer and these holes might be disappeared in case of low percentage of crosslink content in the blend point out that these uncross-linked contents might get swelled in the solvent present in the blend as shown in the figure (Fig. 1b).

\subsection{Optimization of SA-based film forming additives}

Table 1 shows the variation in mechanical properties of SA-based films with the addition of various additives. The TS and YM of SP blend films decreased from 22.39 to 6.12 MPa and 94.92 to $23.08 \mathrm{MPa}$, respectively owing to the plasticization of glycerol that reduced the intermolecular attractions and increased the mobility of the film matrix [22-24]. Nonetheless, the EB increased as the plasticized film showed better water permeability [25], flexibility and higher deformation. The existence of glycerol in the blend was beneficial; since this compound not only plasticized the materials but also increased the concentration of available $\mathrm{OH}$ groups, which could also be involved in 
Fig. 1 SEM images with 5000 times magnification of a SPGMM blend before storage and $\mathbf{b}$ SPGMM blend after storage
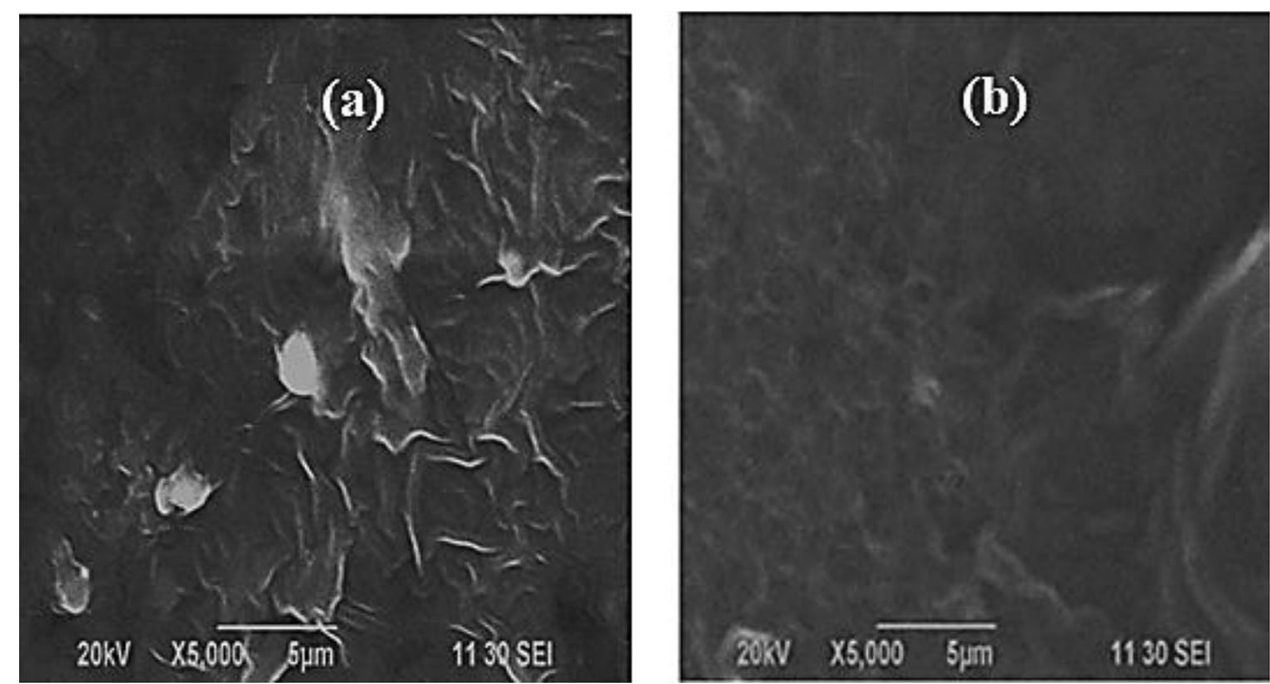

Table 1 Optimization of SA-based film forming additives (wt\%) on the basis of mechanical properties

\begin{tabular}{lccccc}
\hline Tensile properties & S/P (90/10) & $\begin{array}{l}\text { S-P/G } \\
(90-10 / 15)\end{array}$ & $\begin{array}{l}\text { S-P-G/M } \\
(90-10-15 / 20)\end{array}$ & $\begin{array}{l}\text { S-P-G-M/M (90-15-20/7) } \\
10-\end{array}$ & $\begin{array}{r}\text { Radiation } \\
\text { dose@12 kGy }\end{array}$ \\
\hline Tensile strength (MPa) & 22.39 & 6.12 & 5.13 & 33.38 & 43.38 \\
Young modulus (MPa) & 94.92 & 23.08 & 5.56 & 275.99 & 551.29 \\
Elongation at break (\%) & 3.98 & 8.10 & 14.63 & 3.27 & 1.95 \\
\hline
\end{tabular}

transport. It is accepted nowadays that the unique properties of SPGM derive from the nature of not only ionic and covalent bonds, but also hydrogen bonds present in the samples. This type of bonding in the cations and/or anions and especially the interactions occurring between the ions add the mechanical strength to the films. TS and YM of the films further decreased due to incorporation of $\mathrm{MO}$ in the blend and EB increased as that of glycerol. Mustard oil is an emulsifier that acts as binding agent and reduces hydrophilicity. The details of the film forming procedure and results were reported in our previous work [26].

\subsection{Effect of $M A$ addition and $\gamma$ radiation}

Tensile properties of SA-based films of different monomer concentrations as well as $\gamma$ irradiation are shown in Figs. 2, 3 and 4. TS, YM and EB properties of the films with MA (0-15 wt \%) and radiation doses (0-20 kGy) were examined. Radiation doses exhibited significant effect upon the tensile properties with the variation of the concentrations of the monomer which is predominant and clearly observed. Tensile strength and modulus of the formulated films were increased with the increasing of monomer concentrations and radiation doses and decreased the values of elongation at break. The trends were followed up to the monomer concentration of 7 wt $\%$ and radiation dose $12 \mathrm{kGy}$; considered to be optimum and corresponding results are inserted in Table 1.

It has been expected that with the increase in radiation doses, more free radicals can be generated in SA and these radicals may react with MA to form copolymer of alginate and MA results in increased tensile properties of the films. Moreover, free radicals may be formed from the monomer MA which can promote the polymerization reaction. As the monomer concentration increased up to $7 \mathrm{wt} \%$, more MA reacted with SA to give an extended carbon chain. In this situation, cross-linking might occur between the functional groups of methyl acrylate and sodium alginate resulted in both the copolymerization and homopolymerization [27]. At a higher concentration of MA (> $7 \mathrm{wt} \%)$, this could create homopolymer rather than a copolymer backbone because of the dominant recombination process $[27,28]$. In addition, at higher radiation doses (>12 kGy) the films became hard and brittle and possibly degraded [29-32]. Thus, at higher radiation doses, TS and YM of the films decreased, however, EB increased. It can be concluded that at the optimum radiation dose (12 kGy) and MA concentration (7 $w t \%)$, the films became less flexible and gained a minimum EB; this might have been due to the maximum interaction between the polymers. 
Fig. 2 Effect of radiation doses on tensile strength of the SA-based formulated films at different monomer concentrations

Fig. 3 Effect of radiation doses on Young modulus of the SA-based formulated films at different monomer concentrations

\subsection{Effect of storage on mechanical properties}

The effect of storage time on tensile properties of the SAbased films at different radiation doses is presented with standard deviation in Figs. 5, 6 and 7. Figures 5 and 6 show that with the increase of radiation doses, TS and YM of the formulated films increased for both the samples tested before and after storage time results in higher tensile properties (35-80\%) of the stored films. On the contrary, $\mathrm{EB}$ of the stored films for all tested radiation doses were found to be lower values $(<30 \%)$ than those of the irradiated films without storage (Fig. 7) point out the fact that $\gamma$ treated samples increased rigidity of the films causing ultimate drop in EB [29]. At radiation dose of $12 \mathrm{kGy}, \mathrm{TS}$, $\mathrm{YM}$ and $\mathrm{EB}$ of the stored films were $58.56 \mathrm{MPa}, 1011 \mathrm{MPa}$ and $1.38 \%$ and those of non-stored films were $43.38 \mathrm{MPa}$, 
Fig. 4 Effect of radiation doses on elongation at break of the SA-based formulated films at different monomer concentrations

Fig. 5 Effect of storage time on tensile strength of the SA-based formulated films at different radiation doses presented with standard deviation
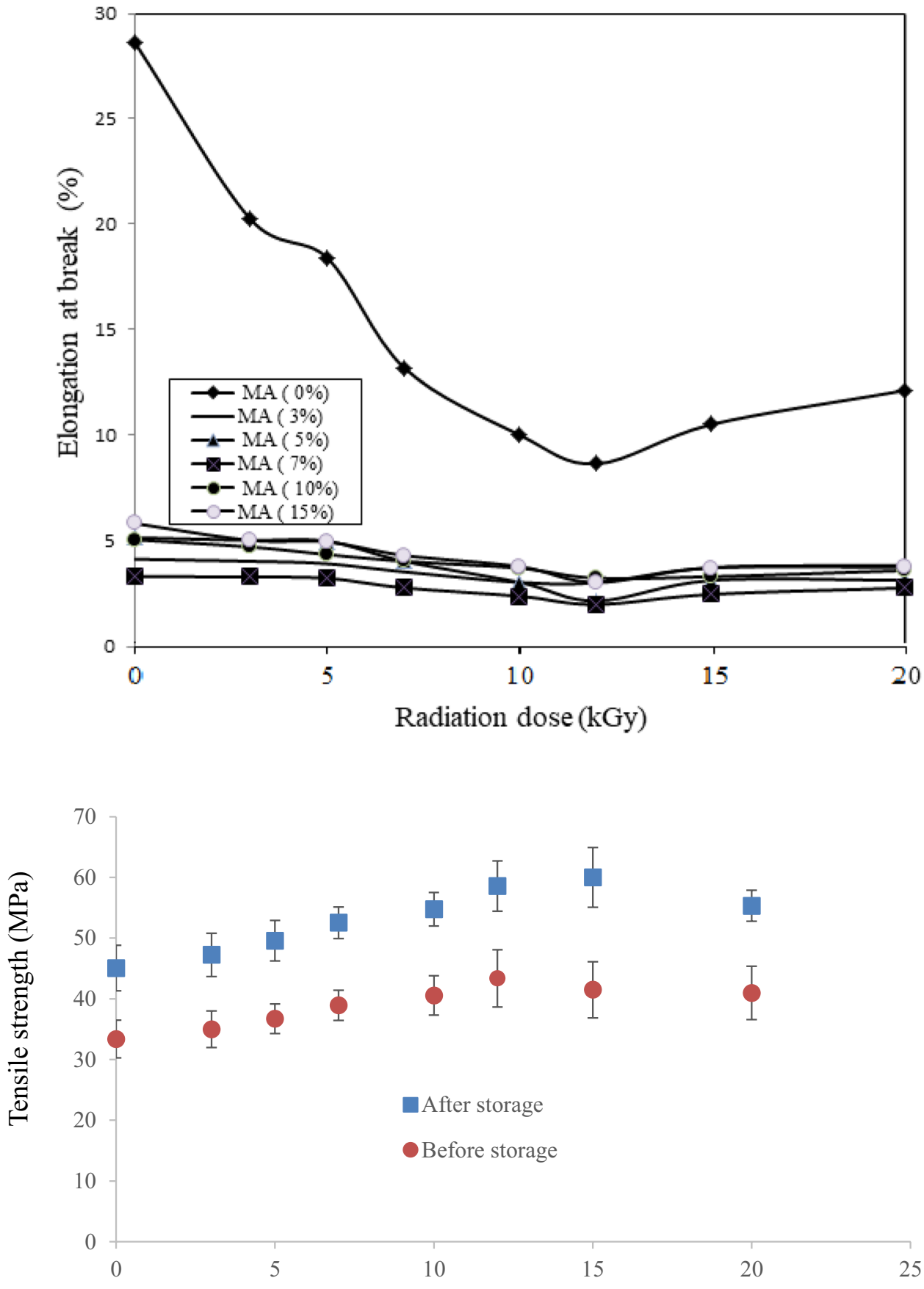

Radiation dose (kGy)
$551.29 \mathrm{MPa}$ and $1.95 \%$, respectively. After that (> $12 \mathrm{kGy}$ ) the values of TS and YM started to decrease and EB exhibited gradual increase for both the stored and non-stored films reported earlier $[26,32,33]$. The reason behind the altered mechanical properties of the stored films might be reformed internal structure (intra-particle diffusion) of the polymer films and restricted oxygen and moisture interaction with the blends in the desiccator [34]. Moreover, stored samples got enough time for cross-linking as well as promoted homopolymerization [26]. A similar behavior was also observed in starch-based films examined at different temperatures [35].

\subsection{DMA analysis}

The viscoelasticity and glass-transition phenomena of the polymeric materials are important factors for investigating dynamic mechanical properties of the films. DMA can be 
Fig. 6 Effect of storage time on Young modulus of the SA-based formulated films at different radiation doses demonstrated with standard deviation
Fig. 7 Effect of storage time on elongation at break of the SAbased formulated films at different radiation doses shown with standard deviation

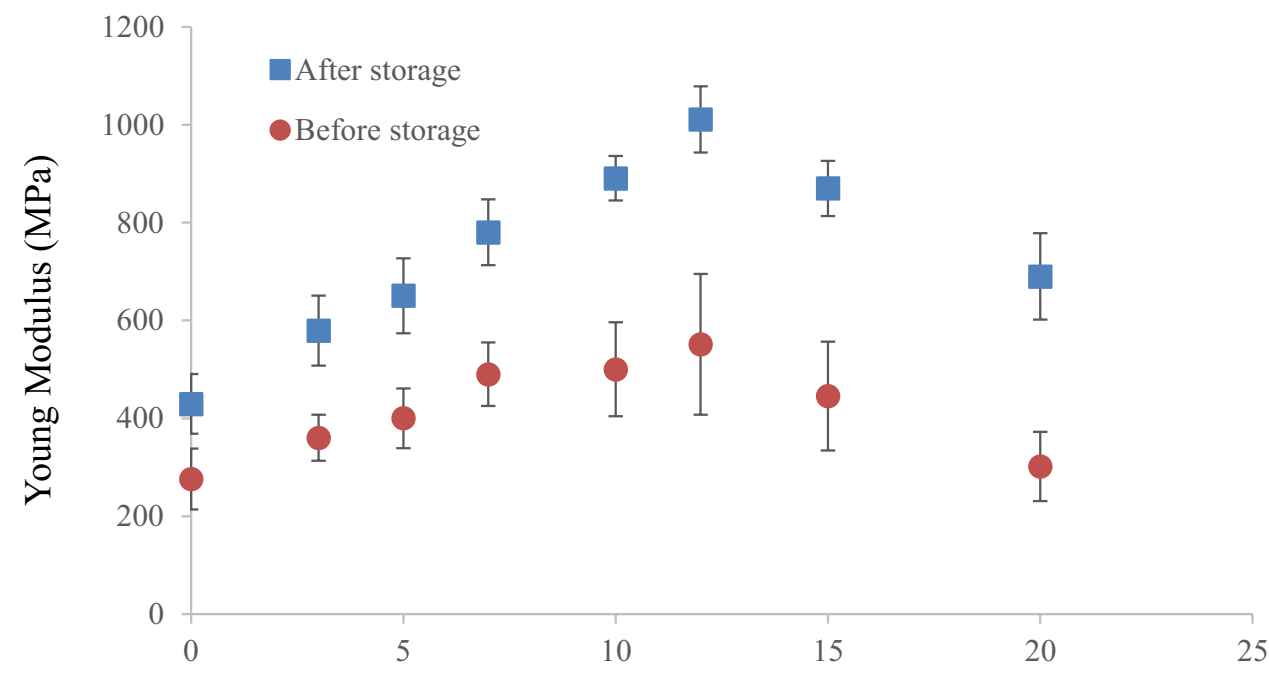

Radiation dose (kGy)

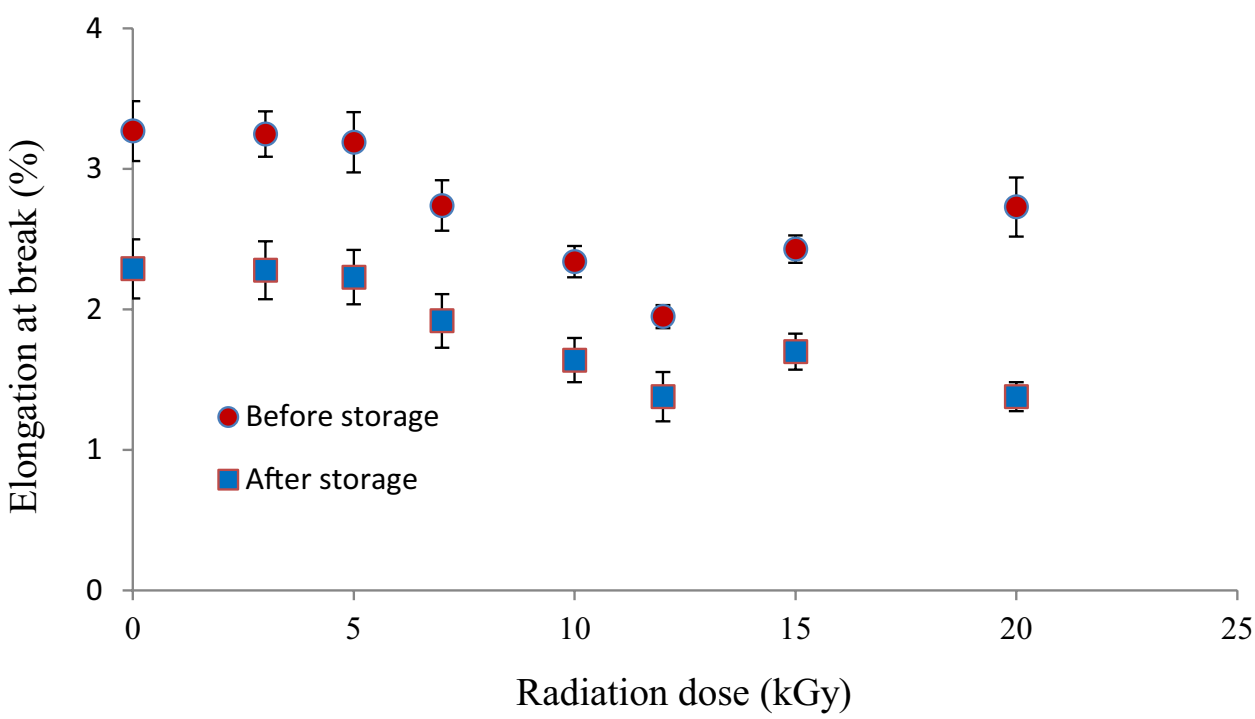

used to explore the glass-transition temperature $\left(T_{g}\right)$ and frequency-dependent nature of the transition. Therefore, DMA was used to examine the behavior of the alginate films in this study.

Figure 8 shows the change in the dynamic moduli of the SPGMM films before and after storage with temperature range 28 to $200^{\circ} \mathrm{C}$. Both curves showed two distinct peaks: one at lower temperature (centered at $45-65^{\circ} \mathrm{C}$ ) and the other at higher temperature (centered at $150-165^{\circ} \mathrm{C}$ ). Moreover, drops in moduli were observed in both of the cases. The modulus of the SPGMM films after storage increased up to $\sim 65^{\circ} \mathrm{C}$ and then showed a downhill trend. This transition is called a transition observed at the glassy region of the temperature around 38 to $102^{\circ} \mathrm{C}$. The alginate films showed two-step transitions: the first transition of the films at a lower temperature was related to its behavior changes because of water evaporation; this was followed by the second transition at a higher temperature, called $T_{g}$, at which the alginate film altered its behavior from glassy to rubbery $\left(155^{\circ} \mathrm{C}\right)$. A sharp drop in the storage modulus was detected in the $\mathrm{T}_{\mathrm{g}}$ region, and in this region, the behavior of alginate films moved to a leathery-state plateau region known as the noncrystalline region caused by the micro-Brownian motion. The modulus of the SPGMM films before storage showed almost similar trend; however, the effect is less prominent.

Three relaxations may be measured by DMA but in this study only a relaxation is discussed that is $>0{ }^{\circ} \mathrm{C}$. The first a relaxation peak might be formed by intra-molecular moisture; second one indicates $T_{g}$ of SA. Caykara et al. reported that melting point of $\mathrm{SA}: \mathrm{PEO}=9: 1(\mathrm{w} / \mathrm{w})$ blend films is $64^{\circ} \mathrm{C}$ [36] and $\mathrm{T}_{\mathrm{g}}$ of pure $\mathrm{SA}$ is $158^{\circ} \mathrm{C}$ [37]. The most 
Fig. 8 DMA plot of the storage modulus versus temperature for the SPGMM films before and after storage

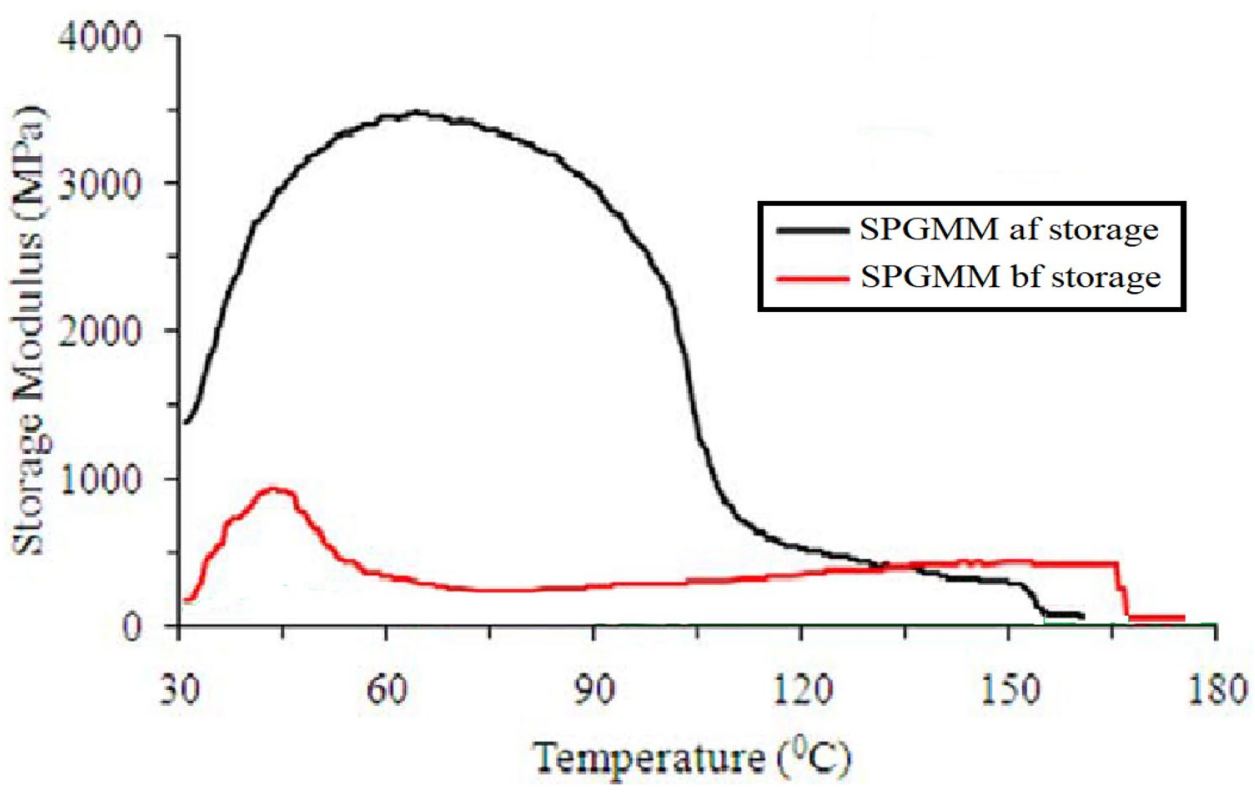

important outcome of the DMA studies was that the first transition of the film increased after storage.

In addition, thermal stability of the SPGMM films is an important criterion towards actual uses of the films. We analyzed TGA and DSC of the films and reported in our previous work that the property of the films was improved by $\gamma$ radiation and $M A$ addition [26]. The thermal degradation of the grafted copolymer occurred at higher temperatures $\left(250-390^{\circ} \mathrm{C}\right)$. Side chains of the polymeric backbone might be eliminated at about $290-460^{\circ} \mathrm{C}$ and the backbone of the films breakdown at $500-570^{\circ} \mathrm{C}$. Almost similar results were also reported for DSC analysis. Polymeric grafting of MA to alginate influenced its thermal behavior as well as increased its hydrophilicity. It can be anticipated from the study that the thermal stability of the SPGMM films would be remarkably increased after storage as the tensile properties of the films was improved exposed above. Therefore, the SA-PEO-Gol-MO-MA films could be safely used from a practical viewpoint.

\subsection{Effect of storage on moisture absorption}

Moisture absorption capacity of the stored and non-stored films was studied for $24 \mathrm{~h}$ at room temperature $\left(25^{\circ} \mathrm{C}\right)$ and average relative humidity of $78 \%$ and the results are presented in Fig. 9. The samples showed two peaks: one at
Fig. 9 Effect of storage time (0-24 h) on moisture absorption of the SA-based formulated films presented with standard deviation

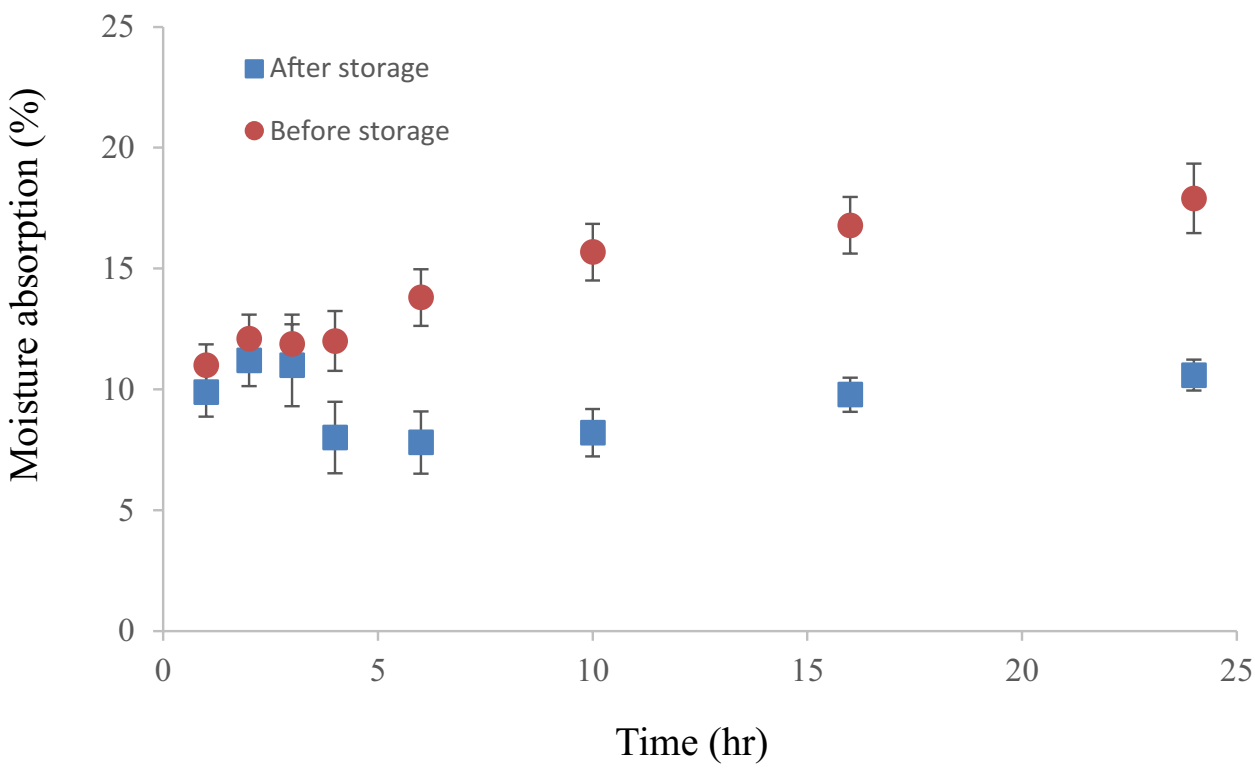


shorter contact/immersion period ( $\sim 2 \mathrm{~h}$, more intense) and the other at longer period (5-20 h) demonstrated the maximum absorption capacity. It was observed that stored films showed lower moisture absorption capacity (10.59\%) than non-stored (17.9\%) films for $20 \mathrm{~h}$ immersion period. The results are statistically satisfactory due to insignificant differences in standard deviation error bars. In our previous work, we reported the effect of mustard oil, methyl acrylate monomer, radiation doses, etc. on the SA-based films [26]. In fact, when the films were modified with MA and $\gamma$-radiation doses, the moisture/vapor content values were lower down due to monomer grafting (copolymerization) reaction that reduced the permeability of water to the films [32]. The moisture absorption capacity of the stored films is substantially lower than that of non-stored films because the rest of the unreacted $\mathrm{OH}$ groups in SA might be reacted with double bond in MA during storage [38]. It was also observed that water uptake values of the films started to decrease with increasing immersion time; which might be due to the degradation of the swollen films. Yi He investigated the moisture absorption and desorption properties of a material by thermogravimetric and dynamic mechanical analysis in a humidity-controlled environment and reported that the moisture diffusion of the material could be described by Fick's diffusion law [39]. However, materials can suffer damage at high temperatures and humidity levels in numerous ways: swelling, reduction in $\mathrm{T}_{\mathrm{g}}$ and physico-mechanical properties of the films. Moreover, particle size, nature of the ions, ionic strength, molecular weight, $\mathrm{pH}$, etc. are the key factors for moisture uptake into the polymeric structures $[4,17]$.

\subsection{Mechanism of storage film formation}

Reaction scheme of irradiated polymeric blend of SPGMM formation during storage is presented in Fig. 10. Sodium alginate based cross-linked polymer films were prepared by casting method. SA with various additives (PEO, Gol and MO) as optimized above reacted with MA monomer exposed to $\gamma$ irradiation prior to storage. The irradiated blend was stored for six months results in degree of cross-linking after natural evaporation of unreacted additives present in the system. Finally SAbased polymer films were obtained and characterized en route for further application.

The as-prepared SPGMM blend was a subjected to drying at room temperature. The mass of the blend changes with time due to the loss of solvent from the blend into the surrounding medium. As the time passes, the amount of solvent on the top decreases and drying rate starts falling due to slow diffusion of solvent from the blends to the blend-air interface [1]. The transport of solvent in polymer films is affected by unsaturation, degree of crystallinity, cross-link density and $\mathrm{T}_{g}$ [40].

In fact, SA-based films might be broken down into smaller molecules other than polymerization during $\mathrm{\gamma}$-irradiation [4]. The most probable cause behind the higher mechanical properties of the stored films is some unreacted functional groups or free radicals present in the polymeric blends which get enough time for various intra-molecular bond formation during the storage period as discussed earlier.

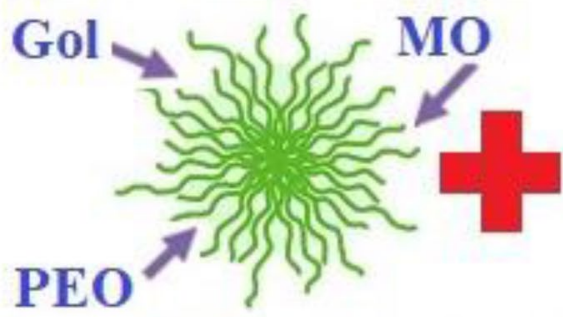

\section{Sodium alginate}
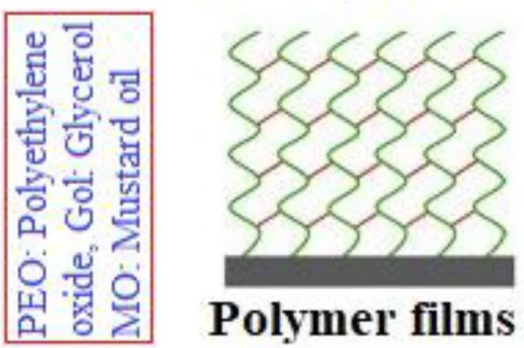
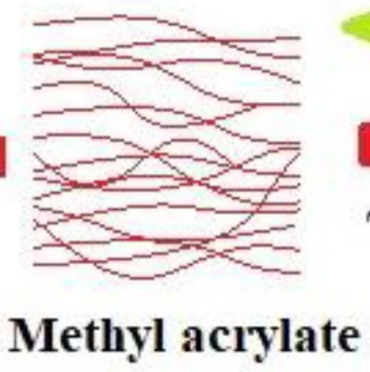

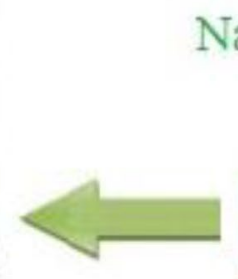

Natural evaporation

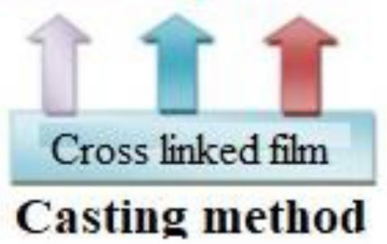

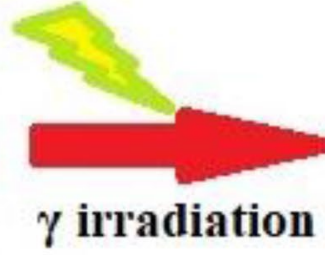

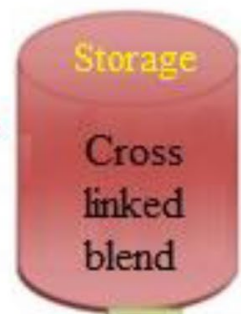

blend

Fig. 10 Schematic presentation of irradiated cross-linked polymeric blend of SPGMM formation during storage 


\subsection{Photodegradation}

Synthetic polymers degrade when they get exposed to UV radiation due to the complex photochemical process. The weather conditions like moisture, temperature and pollutants, etc. enhance this degradation process. Moreover, improper drying and polymerization with other reacting species or ingredients are the main causes of degradation. However, pure polymers hardly degrade because they do not absorb sufficient radiations greater than wavelength of $295 \mathrm{~nm}$ [41]. Moreover, linear polymers having a low degree of cross-linking, might take very long time due to low diffusion coefficient to get the homogeneity unlike to the present issue of cross-linked polymers might be faster owing to the effect of the diffusion coefficient value to a larger extent [42].

In addition, UV absorbers and free radical scavengers are chemically destroyed during weathering process [43, 44]. However, in the present attempt various additives were employed in the SA/PEO blend for different persistence; however, they might have stabilizing and binding properties to improve resistance to weather conditions.

\section{Conclusions}

The effect of polymeric blending and storage time on the physico-mechanical properties of the SA-based PEO films modified by MA monomer and $\gamma$-irradiation were quite successfully presented in the present issue. At optimized radiation dose of $12 \mathrm{kGy}$, TS and YM of the films stored for six months were found to be 58.56 and $1011 \mathrm{MPa}$ and that of non-stored films were obtained $35-80 \%$ because previous samples got enough time for crosslinking as well as promoted homopolymerization. On the contrary, EB of the SPGMM stored films for all tested radiation doses was obtained to be lower values $(\sim 30 \%)$ than that of the irradiated films without storage. Similarly, stored films showed lower moisture absorption capacity $(10.59 \%)$ than that of non-stored (17.9\%) films for an immersion period of $20 \mathrm{~h}$. The $T_{g}$ of SA-based films obtained by DMA test was found to be $\sim 155^{\circ} \mathrm{C}$. The reaction scheme of irradiated polymeric blend of SPGMM formation during storage and its resistance to photodegradation is demonstrated in the manuscript.

\section{Compliance with ethical standards}

Conflict of interest The authors declare that they have no conflict of interest.

\section{References}

1. Pathania A, Sharma J, Arya et al (2018) Effect of crosslinked polymer content on drying of binary polymer-solvent coatings. Prog Org Coat 114:78-89

2. Phupoksakul T, Leuangsukrerk M (2016) Effect of storage temperature and time on stability of poly (lactide)-whey protein isolate laminated films. J Appl Polym Sci 133(16):43547

3. Gacesa P (1988) Alginates. Carbohydr Polym 8(3):161-182

4. Mollah MZI, Khan MA, Khan RA (2009) Effect of gamma irradiated sodium alginate on red amaranth (Amaranthuscruentus L.) as growth promoter. Radiat Phys Chem 78:61-64

5. Hirano S, Mizutani C, Yamaguchi et al (1978) Formation of the polyelectrolyte complexes of some acidic glycosaminoglycans with partially $\mathrm{N}$-acylated chitosans. Biopolymers 17:805-810

6. Hari PR, Chandy T, Sharma CP (1996) Chitosan/calciumalginate beads for oral delivery of insulin. J Appl Polym Sci 59(11):1795-1801

7. Patel GM, Patel CP, Trived HC (1999) Ceric-induced grafting of methyl acrylate onto sodium salt of partially carboxymethylated sodium alginate. Eur Polym J 35:201-208

8. Bailey FE, Koleske JV (1976) Poly (ethylene oxide). Academic Press, New York

9. Abera G, Woldeyes B, Demash et al (2020) The effect of plasticizers on thermoplastic starch films developed from the indigenous Ethiopian tuber crop Anchote (Coccinia abyssinica) starch. Int J Biol Macromol 155:581-587

10. Desai NP, Hubbell JA (1991) Solution technique to incorporate polyethylene oxide and other water-soluble polymers into surfaces of polymeric biomaterials. Biomaterials 12:144-153

11. Desai NP, Hubbell JA (1992) Surface physical interpenetrating networks of poly (ethylene terephthalate) and poly (ethylene oxide) with biomedical applications. Macromolecules 25:226-232

12. Lee JH, Kopecek J, Andrade JD (1989) Protein-resistant surfaces prepared by PEO-containing block copolymer surfactants. J Biomed Mater Res 23:351-368

13. Kondo T, Sawatari C (1994) Intermolecular hydrogen bonding in cellulose/poly(ethylene oxide) blends: thermodynamic examination using 2,3-di-O- and 6-O-methylcelluloses as cellulose model compounds. Polymer 35(20):4423-4428

14. Hendrix KM, Matthew JM, Hahn-Bit et al (2012) Defatted mustard seed meal-based biopolymer film development. Food Hydrocolloids 26(1):118-125

15. Kargarzadeh $\mathrm{HJ}$, Huang N, Lin et al (2018) Recent developments in nanocellulose-based biodegradable polymers, thermoplastic polymers, and porous nanocomposites. Prog Polym Sci 87:197-227

16. Nordin N, Othman SH, Rashid SA, Basha RK (2020) Effects of glycerol and thymol on physical, mechanical, and thermal properties of corn starch films. Food Hydrocolloids 106:105884

17. McHugh TH, Krochta JM (1994) Sorbitol-vs glycerol-plasticized whey protein edible films: integrated oxygen permeability and tensile property evaluation. J Agric Food Chem 42:841-845

18. Kang HJ, Min SC (2010) Potato peel-based biopolymer film development using high-pressure homogenization, irradiation, and ultrasound. Food Sci Technol 43:903-909

19. Yang JM, Wang NC, Chiu et al (2014) Preparation and characterization of poly (vinyl alcohol)/sodium alginate blended membrane for alkaline solid polymer electrolytes membrane. J Mem Sci 457:139-148

20. Chowhan ZT, Amaro AA, Chi LH (1982) Comparative evaluations of aqueous film coated tablet formulations by high humidity aging. Drug Dev Ind Pharm 8(5):713-737 
21. Dafader NC, Rahman ST, Rahman et al (2016) Preparation of gelatin/poly(vinyl alcohol) film modified by methyl methacrylate and gamma irradiation. Int J Polym Anal Charact 21(6):513-523

22. Bravo-Osuna I, Ferrero C, Jimenez-Castellanos MR (2005) Water sorption-desorption behaviour of methyl methacrylate-starch copolymers: effect of hydrophobic graft and drying method. J Eur J Pharm Biopharm 59(3):537-548

23. Sothornvit R, Krochta JM (2000) Plasticizer effect on oxygen permeability of $\beta$-lactoglobulin films. J Agric Food Chem 48(12):6298-6302

24. Rodriguez M, Oses J, Ziani et al (2006) Combined effect of plasticizers and surfactants on the physical properties of starch based edible films. Food Res Int 39(8):840-846

25. Gontard N, Guilbert S, Cuq JL (1993) Water and glycerol as plasticizers affect mechanical and water vapor barrier properties of an edible wheat gluten film. J Food Sci 58(1):206-211

26. Sumi MSA, Rahman MW, Alam et al (2016) Irradiated sodiumalginate/polyethylene oxide blend films Improved by methyl acrylate monomer. J Appl Polym Sci 133(26):43562

27. Alam R, Khan MA, Khan et al (2008) Study on the physicomechanical properties of photo-cured chitosan films with oligomer and acrylate monomer. J Polym Environ 16(3):213-219

28. Nunthanid J, Puttipipatkhachorn S, Yamamoto et al (2001) Physical properties and molecular behavior of chitosan films. Drug Dev Ind Pharm 27(2):143-157

29. Rahman MW, Haque ME, Rahman et al (2009) Improvement of physico-mechanical properties of radiation vulcanised natural rubber latex films by adding urea. Polym Plast Technol Eng 48:696-700

30. Zelinska K, Shostenko AG, Truszkowski S (2009) Radiolysis of chitosan. High Energy Chem 43:445-448

31. Rahman MW, Hossain MM, Alam et al (2013) Addition of transition metals to improve physico-mechanical properties of radiation-vulcanized natural rubber latex films. Int J Polym Anal Chem 18:479-487

32. Tuhin MO, Rahman N, Haque et al (2012) Modification of mechanical and thermal property of chitosan-starch blend films. Radiat Phys Chem 81(10):1659-1668

33. Dafader NC, Haque ME, Akhtar F (2005) Variation of physicchemical properties of radiation crosslinked rubber with storage time. J Polym Sci 23(5):497-501
34. Chow MJ, Zhang Y (2011) Changes in the mechanical and biochemical properties of aortic tissue due to cold storage. J Surg Res 171(2):434-442

35. Palma-Rodríguez HM, Berrios JDJ, Glenn et al (2016) Effect of the storage conditions on mechanical properties and microstructure of biodegradable baked starch foams. CyTA J Food 14(3):415-422

36. Caykara T, Demirci S, Eroğlu et al (2005) Poly (ethylene oxide) and its blends with sodium alginate. Polymer 46(24):10750-10757

37. Roger S, Bee A, Balnois et al (2004) Presented at the fifth international conference on polymer-solvent complexes and intercalates, Lorient, France

38. Morton WE, Hearle JWS (1962) Physical Properties of Textile Fibers. Manchester and London, The Textile Institute and Butterworths. pp xxi +608

39. Yi He (2012) Moisture absorption and hygroscopic swelling behavior of an underfill material. Thermochim Acta 546:143-152

40. George SC, Thomas S (2001) Transport phenomena through polymeric systems. Prog Polym Sci 26:985-1017

41. Nguyen T, Gu X, Vanlandingham et al (2013) Degradation modes of crosslinked coatings exposed to photolytic environment. J Coat Technol Res 10:1-14

42. Lipatov Y, Chornaya V, Nesterov et al (1984) Concentration dependence of thermodynamic interaction parameters in a polymer-polymer-solvent system. Polym Bull 12:49-53

43. Peters C, Ellwood K, Srivastava et al (2007) Ultraviolet light absorber mobility in crosslinked coatings: experiments and modeling. Prog Org Coat 58:272-281

44. Pathania A, Arya RK, Ahuja S (2017) Crosslinked polymeric coatings: preparation, characterization, and diffusion studies. Prog Org Coat 105:149-162

Publisher's Note Springer Nature remains neutral with regard to jurisdictional claims in published maps and institutional affiliations. 